July 4, 2018

\title{
Quantization of Drinfel'd doubles
}

\author{
A. Ballesteros ${ }^{1}$, E. Celeghini ${ }^{2}$ and M.A. del Olmo ${ }^{3}$ \\ ${ }^{1}$ Departamento de Física, Universidad de Burgos, \\ E-09006, Burgos, Spain. \\ ${ }^{2}$ Departimento di Fisica, Universitá di Firenze and INFN-Sezione di Firenze \\ I50019 Sesto Fiorentino, Firenze, Italy \\ ${ }^{3}$ Departamento de Física Teórica, Universidad de Valladolid, \\ E-47011, Valladolid, Spain. \\ e-mail: angelb@ubu.es, celeghini@fi.infn.it,olmo@fta.uva.es
}

\begin{abstract}
Hopf algebra quantizations of 4-dimensional and 6-dimensional real classical Drinfel'd doubles are studied by following a direct "analytic" approach. The full quantization is explicitly obtained for most of the Drinfel'd doubles, except a small number of them for which the dual Lie algebra is either $\mathfrak{s l}_{2}$ or $\mathfrak{s o}(3)$. In the latter cases, the classical $r$-matrices underlying the Drinfel'd double quantizations contain known standard ones plus additional twists. Several new four and six-dimensional quantum algebras are presented and some general features of the method are emphasized.
\end{abstract}

MSC: 81R50, 81R40, 17B37

Keywords: Lie bialgebras, Drinfel'd double, $r$-matrix, quantization, quantum algebras 


\section{Introduction}

The essential role that Lie bialgebras play in the quantization of Poisson-Lie structures comes from the well-known result by Drinfel'd [1 that establishes a one to one correspondence between Poisson-Lie groups and Lie bialgebras. In fact, the concept of classical double [1] is just a reformulation of that of Lie bialgebra in terms of a (double) dimensional Lie algebra endowed with a suitable pairing. This "duplication" process can be iterated by taking into account that the double Lie algebra can be in turn equipped with a quasitriangular Lie bialgebra structure by means of a canonical classical $r$-matrix.

The Hopf algebra quantization of such a double Lie bialgebra is the so-called quantum double, a basic object in quantum group theory (see, for instance, [2]-9] for a detailed exposition and references therein). In particular, quantum doubles are helpful for the explicit construction of quantum $R$-matrices of quantum groups and supergroups by following a "universal $\mathcal{T}$-matrix approach" [10] (see [1]-[17]) and have been also considered as symmetries in quantum field theory [18, 19]. Moreover, $\sigma$-models related by Poisson-Lie T-duality are directly connected with classical doubles (see [20]-22] and references therein).

We also recall that in Ref. 23] the full classification of two and three dimensional (3D) real Lie bialgebra structures and their associated Drinfel'd doubles has been obtained. That result is tantamount to the (first order) classification of quantum deformations of $2 \mathrm{D}$ and 3D real Lie algebras. Complementarily, the classification of 4D and 6D Drinfel'd doubles has been performed in [22] and [24], respectively, by following a "direct" approach. Throughout the paper we will preserve the notation and labeling for the Lie bialgebras and Drinfel'd doubles given in [23, although both classifications are fully equivalent (up to the fact that 23. does not consider $6 \mathrm{D}$ classical doubles coming from a trivial Lie bialgebra with vanishing cocommutator $\delta$ or, equivalently, with abelian dual $\mathfrak{g}^{*}$ ).

The aim of this work is to provide a global insight into the Hopf algebra quantization of 4D and 6D Drinfel'd doubles (DD) by making use of a direct quantization procedure that has been recently used in order to obtain and classify 3D quantum algebras 25]. The motivation for this study is two-fold.

Firstly, the family of 6D DD algebras is physically interesting. For instance, it contains the $s o(3,1)$ and $s o(2,2)$ algebras as well as the $(2+1)$ Poincaré algebra. Therefore the DD quantizations will provide quantum deformations for these algebras. In particular, the class of DDs with $\mathfrak{g}=\mathfrak{r}_{3}(1)$ can be thought as $s o(p, q)$ Lie algebras (with $p+q=4$ ) and some of their contractions [26]. In general, such quasitriangular quantizations turn out to be superpositions of a standard (quasitriangular) quantization plus twists. We will discuss the properties of all these quantum algebras and we explicitly obtain some of them by making use of a quantization procedure recently introduced by us [25].

Secondly, since Lie bialgebras (therefore, DD algebras) provide a classification of firstorder quantum deformations, the set of DD quantizations can be considered as an appropriate setting for the search of classification schemes for quantum deformations. In fact, several strong regularities are found within the set of quantum algebras that are presented in this work, and these common facts can be thought of guidelines for a future research program of a classification "á la Cartan" of quantum groups [25]. Among them, we mention the following 
common properties of the quantizations:

- All 4D and 6D DD algebras are non-simple Lie algebras.

- The only functions appearing in the deformed commutation rules and coproducts are exponentials and polynomials [27].

- The quantization can be obtained by following a "analytic approach" in the full symmetrized basis of the quantum universal enveloping algebra that preserves a "generalized cocommutativity" property [25].

- All the DD deformations coming from the canonical skew-symmetric DD $r$-matrix are standard ones.

- The "crossed" commutation rules between the algebra and the dual contain the most complex part of the quantum deformations.

The structure of the paper is as follows: Section 2 is devoted to fix the notation. Section 3 presents the study of quantum 2D DD algebras as a toy-model for further quantizations. The following sections develop the quantizations of 6D DD algebras by following the classification given in 23]. Finally, some remarks close the paper.

\section{Drinfel'd Double (bi)algebras}

Let us consider a Lie bialgebra $(\mathfrak{g}, \delta)$ and a basis $\left\{x^{i}\right\}$ of $\mathfrak{g}$. Such a Lie bialgebra can be characterized by a pair of structure tensors $\left(f_{n}^{l m}, c_{i j}^{k}\right)$, i.e.,

$$
\left[x^{i}, x^{j}\right]=f_{k}^{i j} x^{k}, \quad \delta\left(x^{n}\right)=c_{l m}^{n} x^{l} \otimes x^{m} .
$$

In this language, the cocycle condition for the cocommutator $\delta$ becomes the following compatibility condition between the tensors $c$ and $f$

$$
f_{k}^{a b} c_{i j}^{k}=f_{i}^{a k} c_{k j}^{b}+f_{i}^{k b} c_{k j}^{a}+f_{j}^{a k} c_{i k}^{b}+f_{j}^{k b} c_{i k}^{a} .
$$

Now we fix a basis $\left\{X_{i}\right\}$ for the dual algebra $\mathfrak{g}^{*}$ through the following pairing

$$
\left\langle X_{i}, X_{j}\right\rangle=0, \quad\left\langle x^{i}, x^{j}\right\rangle=0, \quad\left\langle x^{i}, X_{j}\right\rangle=\delta_{j}^{i}, \quad \forall i, j .
$$

Then $\left(\mathfrak{g}^{*}, \eta\right)$ is also a Lie bialgebra with structure tensors $(f, c)$, i.e.,

$$
\left[X_{i}, X_{j}\right]=c_{i j}^{k} X_{k}, \quad \eta\left(X_{n}\right)=f_{n}^{l m} X_{l} \otimes X_{m}
$$

This duality leads to the consideration of the pair $\left(\mathfrak{g}, \mathfrak{g}^{*}\right)$ and its associated vector space $\mathfrak{g} \oplus \mathfrak{g}^{*}$, that can be endowed with a Lie algebra structure by means of the commutators

$$
\left[x^{i}, x^{j}\right]=f_{k}^{i j} x^{k}, \quad\left[X_{i}, X_{j}\right]=c_{i j}^{k} X_{k}, \quad\left[x^{i}, X_{j}\right]=c_{j k}^{i} x^{k}-f_{j}^{i k} X_{k}
$$


This Lie algebra, $D(\mathfrak{g})$, is called the Double Lie algebra of $(\mathfrak{g}, \delta)$. Obviously, $\mathfrak{g}$ and $\mathfrak{g}^{*}$ are subalgebras of $D(\mathfrak{g})$, and the compatibility condition (10) is just the Jacobi identity for (2).

Moreover, if $\mathfrak{g}$ is a finite dimensional Lie algebra, then $D(\mathfrak{g})$ can be endowed with a (quasitriangular) Lie bialgebra structure $\left(D(\mathfrak{g}), \delta_{D D}\right)$ generated by the classical $r$-matrix

$$
r=\sum_{i} x^{i} \otimes X_{i}
$$

or, equivalently, by its skew-symmetric counterpart

$$
\tilde{r}=\frac{1}{2} \sum_{i} x^{i} \wedge X_{i} .
$$

In this respect, note that

$$
C=\sum_{i}\left(x^{i} X_{i}+X_{i} x^{i}\right)
$$

is always a Casimir operator for the DD algebra. Hence, if we denote

$$
\Omega=\sum_{i}\left(x^{i} \otimes X_{i}+X_{i} \otimes x^{i}\right)
$$

then $[1 \otimes Y+Y \otimes 1, \Omega]=0$ for any generator $Y$ of the DD algebra and $\tilde{r}=r-\frac{1}{2} \Omega$.

The cocommutator $\delta_{D D}$ derived from (3) is

$$
\delta_{D D}\left(x^{i}\right)=\delta\left(x^{i}\right)=c_{j k}^{i} x^{j} \otimes x^{k} \quad \delta_{D D}\left(X_{i}\right)=-\eta\left(X_{i}\right)=-f_{i}^{j k} X_{j} \otimes X_{k} .
$$

In fact this "double Lie bialgebra" has as sub-Lie-bialgebras the original one $(\mathfrak{g}, \delta)$ and its dual $\left(\mathfrak{g}^{*}, \eta\right)$.

\section{Quantum two-dimensional DD algebras}

In this section we shall illustrate the procedure that we are going to follow in the rest of the paper by considering the quantization of the 2D DD algebras. In general, we shall proceed as follows:

1. We obtain a quantum coproduct and deformed commutation rules for each of the Hopf subalgebras that quantize the sub-Lie-bialgebras $(\mathfrak{g}, \delta)$ and $\left(\mathfrak{g}^{*}, \eta\right)$.

2. We find the deformed counterparts for the "crossed" commutation rules $\left[x^{i}, X_{j}\right]$.

We will use the Gomez results and notation for Lie bialgebras [23, that in the twodimensional case are shown in Table I. In the first row the Lie sub-Lie-bialgebra structure of 2D DD algebras is described, and their Lie algebra brackets are explicitly given in the most appropriate basis. 
Table I. Two-dimensional DD algebras.

\begin{tabular}{|c|c|c|}
\hline & $\mathfrak{b}_{2} \odot R^{2}$ & $g l(2)$ \\
\hline \hline$\left[x^{0}, x^{1}\right]$ & $x^{1}$ & $x^{1}$ \\
\hline$\left[X_{0}, X_{1}\right]$ & $X_{0}$ & $\lambda X_{1}$ \\
\hline$\left[x^{0}, X_{0}\right]$ & $x^{1}$ & 0 \\
\hline$\left[x^{0}, X_{1}\right]$ & $-x^{0}-X_{1}$ & $-X_{1}$ \\
\hline$\left[x^{1}, X_{0}\right]$ & 0 & $\lambda x^{1}$ \\
\hline$\left[x^{1}, X_{1}\right]$ & $X_{0}$ & $-\lambda x^{0}+X_{0}$ \\
\hline
\end{tabular}

In this case there are only two DD algebras, the "standard one" (isomorphic to a semidirect product of the Borel subalgebra $\mathfrak{b}_{2}$ and $R^{2}$, hereafter denoted as $\mathfrak{b}_{2} \odot R^{2}$ ) and the "non-standard one" (with $\lambda \neq 0$ ), which is isomorphic to $g l(2)$. Let us now describe their quantization (see 24] for the connection of both DD algebras with with Poisson-Lie Tduality).

\subsection{The case $\mathfrak{b}_{2} \odot R^{2}$}

A coproduct for this quantum DD algebra with the "generalized cocommutativity" property 25] would be:

$$
\begin{array}{ll}
\Delta\left(x^{0}\right)=e^{-z x^{1}} \otimes x^{0}+x^{0} \otimes e^{z x^{1}}, & \Delta\left(X_{0}\right)=1 \otimes X_{0}+X_{0} \otimes 1, \\
\Delta\left(x^{1}\right)=1 \otimes x^{1}+x^{1} \otimes 1, & \Delta\left(X_{1}\right)=e^{-z X_{0}} \otimes X_{1}+X_{1} \otimes e^{z X_{0}} .
\end{array}
$$

Note that this coassociative coproduct has to be defined in such a way that the dual of the first-order deformation in the deformation parameter $z$ for the $\left\{x^{0}, x^{1}\right\}$ (resp. $\left\{X_{0}, X_{1}\right\}$ ) generators provides the dual Lie algebra $\left\{X_{0}, X_{1}\right\}$ (resp. $\left\{x^{0}, x^{1}\right\}$ ). Explicitly,

$$
\Delta=\Delta_{0}+z \delta_{D D}+o\left[z^{2}\right]
$$

where $\Delta_{0}(y)=1 \otimes y+y \otimes 1$ is the primitive coproduct.

The quantum commutation rules compatible with the previous coproduct (and such that they deform the Table I relations) can be split into two subsets:

Quantum subalgebras:

$$
\left[x^{0}, x^{1}\right]=\frac{\sinh \left(z x^{1}\right)}{z}, \quad\left[X_{0}, X_{1}\right]=\frac{\sinh \left(z X_{0}\right)}{z} .
$$

Quantum crossed relations:

$$
\begin{array}{ll}
{\left[x^{0}, X_{0}\right]=\frac{\sinh \left(z x^{1}\right)}{z},} & {\left[x^{1}, X_{0}\right]=0,} \\
{\left[x^{0}, X_{1}\right]=-\cosh \left(z X_{0}\right) x^{0}-\cosh \left(z x^{1}\right) X_{1},} & {\left[x^{1}, X_{1}\right]=\frac{\sinh \left(z X_{0}\right)}{z} .}
\end{array}
$$

To our knowledge, this is a new four-generators quantum algebra, whose additional central element is $x^{1}-X_{0}$. We also remark that, in spite of appearances, also the right hand side of $\left[x^{0}, X_{1}\right]$ belongs to the symmetric quantum universal enveloping algebra. 


\subsection{The $g l(2)$ case}

The quantization of the remaining DD leads to the following coproduct:

$$
\begin{array}{ll}
\Delta\left(x^{0}\right)=1 \otimes x^{0}+x^{0} \otimes 1, & \Delta\left(X_{0}\right)=1 \otimes X_{0}+X_{0} \otimes 1, \\
\Delta\left(x^{1}\right)=e^{z \lambda x^{0}} \otimes x^{1}+x^{1} \otimes e^{-z \lambda x^{0}}, & \Delta\left(X_{1}\right)=e^{-z X_{0}} \otimes X_{1}+X_{1} \otimes e^{z X_{0}} .
\end{array}
$$

and quantum commutation rules:

Subalgebras

$$
\left[x^{0}, x^{1}\right]=x^{1}, \quad\left[X_{0}, X_{1}\right]=\lambda X_{1}
$$

Crossed relations

$$
\begin{array}{ll}
{\left[x^{0}, X_{0}\right]=0,} & {\left[x^{1}, X_{0}\right]=\lambda x^{1},} \\
{\left[x^{0}, X_{1}\right]=-X_{1},} & {\left[x^{1}, X_{1}\right]=\frac{\sinh \left(z\left(-\lambda x^{0}+X_{0}\right)\right)}{z} .}
\end{array}
$$

We mention that $C=\lambda x^{0}+X_{0}$ is a Casimir operator for this quantum algebra, which has been already studied in [28. Note that the case $\lambda=0$ is isomorphic to the "standard case", as it has been pointed out for the DD algebra in [24].

\section{Quantum DD algebras: $\mathfrak{g}=\mathfrak{r}_{3}(1)$}

In the 3D case we are going to follow the same strategy and notation. For the sake of simplicity, we shall split Gomez's classification of 3D Lie bialgebras into different classes according to the structure of the Lie algebra $\mathfrak{g}$. For each of these classes we shall start by presenting a comprehensive Table of classical results including the pairs of corresponding 3D dual Lie algebras $\left(\mathfrak{g}, \mathfrak{g}^{*}\right)$ (first row), the Lie algebra characterization of the DD algebras (second row) and Gomez's notation 23. for the all the possible Lie bialgebras (third row). Afterwards, the non-deformed Lie brackets are presented by columns in the usual basis (following [23], $\lambda$ is a nonzero essential parameter and $\omega= \pm 1$ ). Next, we shall proceed to the quantization.

We start with the $\mathfrak{g}=\mathfrak{r}_{3}(1)$ cases, that are explicitly given in Table II. We stress that the DDs of type $\left(\mathfrak{r}_{3}(1), \mathfrak{s l}_{2}\right),\left(\mathfrak{r}_{3}(1), \mathfrak{s o}_{3} / \mathfrak{s l}_{2}\right)$ and $\left(\mathfrak{r}_{3}(1), \mathfrak{s l}_{2}\right)$ are the only DDs for which we have not succeeded in obtaining a complete quantization, since we have not been able to construct the quantum coproduct for the $(\mathfrak{g}, \delta)$ sub-Lie-bialgebra. However, we shall see in the sequel that it is possible to identify these quantum algebras as Drinfel'd twists of previously known $\mathfrak{s l}_{2} \oplus \mathfrak{s l}_{2}, \mathfrak{s o}(1,3)$ and $(2+1)$ Poincaré algebra deformations. 
Table II. DD algebras with $\mathfrak{g}=\mathfrak{r}_{3}(1)$.

\begin{tabular}{|c|c|c|c|c|c|c|}
\hline$\left(\mathfrak{g}_{1} \mathfrak{g}^{*}\right)$ & $\left(\mathfrak{r}_{3}(1), \mathfrak{s l}_{2}\right)$ & $\left(\mathfrak{r}_{3}(1), \mathfrak{s o}_{3} / \mathfrak{s l}_{2}\right)$ & $\left(\mathfrak{r}_{3}(1), \mathfrak{s l}_{2}\right)$ & $\left(\mathfrak{r}_{3}(1), \mathfrak{s}_{3}(0)\right)$ & $\left(\mathfrak{r}_{3}(1), \mathfrak{n}_{3}\right)$ & $\left(\mathfrak{r}_{3}(1), \mathfrak{r}_{3}(-1) / \mathfrak{s}_{3}(0)\right)$ \\
\hline $\mathrm{DD}$ & $\mathfrak{s l}_{2} \oplus \mathfrak{s l}_{2}$ & $\mathfrak{s o}(1,3)$ & $\mathfrak{s l}_{2} \odot R^{3}$ & $\mathfrak{s o}(1,3)$ & $\mathfrak{s l}_{2} \odot R^{3}$ & $\mathfrak{s l}_{2} \odot R^{3}$ \\
\hline$[23]$ Nr. & $(1)$ & $(4)$ and $(2)$ & $(3)$ & 9 & 10 & 11 and $11^{\prime}$ \\
\hline \hline$\left[x^{0}, x^{1}\right]$ & $x^{1}$ & $x^{1}$ & $x^{1}$ & $x^{1}$ & $x^{1}$ & $x^{1}$ \\
\hline$\left[x^{0}, x^{2}\right]$ & $x^{2}$ & $x^{2}$ & $x^{2}$ & $x^{2}$ & $x^{2}$ & 0 \\
\hline$\left[x^{1}, x^{2}\right]$ & 0 & 0 & $X_{0}$ & $\lambda X_{2}$ & 0 & 0 \\
\hline$\left[X_{0}, X_{1}\right]$ & $\lambda X_{1}$ & $\lambda X_{2}$ & $X_{1}$ & $-\lambda X_{1}$ & $X_{1}$ & $X_{1}$ \\
\hline$\left[X_{0}, X_{2}\right]$ & $-\lambda X_{2}$ & $-\lambda X_{1}$ & $X_{2}$ & 0 & 0 & $\omega X_{0}$ \\
\hline$\left[X_{1}, X_{2}\right]$ & $X_{0}$ & $\omega X_{0}$ & $x^{1}$ & 0 & 0 & 0 \\
\hline$\left[x^{0}, X_{0}\right]$ & 0 & 0 & $-x^{0}-X_{1}$ & $-X_{1}$ & $-X_{1}$ & $\omega x^{2}-X_{1}$ \\
\hline$\left[x^{0}, X_{1}\right]$ & $x^{2}-X_{1}$ & $\omega x^{2}-X_{1}$ & $-X_{2}$ & $-X_{2}$ & $-\omega x^{1}-X_{2}$ \\
\hline$\left[x^{0}, X_{2}\right]$ & $-x^{1}-X_{2}$ & $-\omega x^{1}-X_{2}$ & $-X_{2}$ & $-X_{2}$ & $x^{2}$ \\
\hline$\left[x^{1}, X_{0}\right]$ & $\lambda x^{1}$ & $-\lambda x^{2}$ & $x^{2}$ & $-\lambda x^{2}$ & $x^{2}$ & $X_{0}$ \\
\hline$\left[x^{1}, X_{1}\right]$ & $-\lambda x^{0}+X_{0}$ & $X_{0}$ & $X_{0}$ & $X_{0}$ & $X_{0}$ & $-x^{0}$ \\
\hline$\left[x^{1}, X_{2}\right]$ & 0 & $\lambda x^{0}$ & $-x^{0}$ & $\lambda x^{0}$ & $-x^{0}$ & 0 \\
\hline$\left[x^{2}, X_{0}\right]$ & $-\lambda x^{2}$ & $\lambda x^{1}$ & 0 & $\lambda x^{1}$ & 0 & $X_{0}$ \\
\hline$\left[x^{2}, X_{1}\right]$ & 0 & $-\lambda x^{0}$ & $x^{2}$ & $-\lambda x^{0}$ & 0 & $X_{0}$ \\
\hline$\left[x^{2}, X_{2}\right]$ & $\lambda x^{0}+X_{0}$ & $X_{0}$ & $-x^{1}+X_{0}$ & $X_{0}$ & $X_{0}$ & \\
\hline
\end{tabular}

\subsection{Type $\left(\mathfrak{r}_{3}(1), \mathfrak{s l}_{2}\right)$}

In order to characterize the classical $r$-matrix of this DD algebra, we could consider the following pairing-preserving change of basis:

$$
\begin{aligned}
\hat{X}_{1} & =\frac{1}{\sqrt{2}}\left(X_{1}+X_{2}\right)+\frac{1}{2 \sqrt{2}}\left(x^{1}-x^{2}\right), \\
\hat{X}_{2} & =\frac{1}{\sqrt{2}}\left(X_{1}-X_{2}\right)-\frac{1}{2 \sqrt{2}}\left(x^{1}+x^{2}\right), \\
x^{\prime} & =\frac{1}{\sqrt{2}}\left(x^{1}+x^{2}\right), \\
x^{\prime 2} & =\frac{1}{\sqrt{2}}\left(x^{1}-x^{2}\right) .
\end{aligned}
$$

Now, if we recall the commutation relations of the Lie algebra $g_{\left(\mu_{1}, \mu_{2}, \mu_{3}\right)}$, a three-parameter family of graded contractions of $s o(2,2)$ [26] (with $\mu_{i}$ being real parameters), we can make the following identification between the DD Lie algebra of type $\left(\mathfrak{r}_{3}(1), \mathfrak{s l}_{2}\right)$ and the $g_{\left(\mu_{1}, \mu_{2}, \mu_{3}\right)}$ generators:

$$
\begin{array}{lll}
N_{3}=2 X_{0}, & J_{3}=2 x^{0}, & J_{+}=-x^{\prime 2}, \\
N_{+}=x^{1}, & J_{-}=-\hat{X}_{1}, & N_{-}=\hat{X}_{2} .
\end{array}
$$

It turns out that the DD algebra $\left(\mathfrak{r}_{3}(1), \mathfrak{s l}_{2}\right)$ is just a $s o(2,2)$ algebra $g_{(\lambda,-1 / 2, \lambda)}$. Since the associated skew-symmetric classical $r$-matrix is $\tilde{r}=\frac{1}{2} \sum_{i} x^{i} \wedge X_{i}$, we get

$$
\tilde{r}=r_{s}+r_{t 1}+r_{t 2}=-\frac{1}{2}\left(N_{+} \wedge J_{-}+J_{+} \wedge N_{-}\right)-\frac{1}{8} N_{3} \wedge J_{3}+\frac{1}{2} N_{+} \wedge J_{+} .
$$

Thus, we have that the quantum DD algebra of type $\left(\mathfrak{r}_{3}(1), \mathfrak{s l}_{2}\right)$ would be isomorphic to the standard deformation of $s o(2,2)$ [26] generated by $r_{s}$ plus two non-commuting twists generated by $r_{t 1}+r_{t 2}=-\frac{1}{8} N_{3} \wedge J_{3}+\frac{1}{2} N_{+} \wedge J_{+}$. 


\subsection{Types $\left(\mathfrak{r}_{3}(1), \mathfrak{s o}_{3} / \mathfrak{s l}_{2}\right)$}

The two cases are labeled by $\omega \lambda$ : when $\omega \lambda>0$ we have the DD algebra $\left(\mathfrak{r}_{3}(1), \mathfrak{s o}_{3}\right)$, and $\omega \lambda<0$ corresponds to $\left(\mathfrak{r}_{3}(1), \mathfrak{s l}_{2}\right)$.

By following the same procedure we perform the change of basis:

$$
\hat{X}_{1}=X_{1}-\frac{\omega}{2} x^{2}, \quad \hat{X}_{2}=X_{2}+\frac{\omega}{2} x^{1}
$$

and we obtain that the DD algebra so $(3,1)$ is isomorphic to the one with parameters $g_{(-\lambda,-1 / 2, \lambda)}$. The associated classical $r$-matrix of this DD algebra is again

$$
\tilde{r}=r_{s}+r_{t 1}+r_{t 2}=-\frac{1}{2}\left(N_{+} \wedge J_{-}+J_{+} \wedge N_{-}\right)-\frac{1}{8} N_{3} \wedge J_{3}-\frac{\omega}{2} N_{+} \wedge J_{+} .
$$

Thus, in this case we would have the standard deformation of $\mathfrak{s o}(1,3)$ [26] plus two noncommuting Drinfel'd twists.

\subsection{Type $\left(\mathfrak{r}_{3}(1), \mathfrak{s l}_{2}\right)$}

Once again, through the following change of basis

$$
\hat{X}_{1}=X_{1}+x^{0}, \quad \hat{X}_{0}=X_{0}-x^{1},
$$

the Lie algebra parameters turn out to be $g_{(0,-1 / 2,1)}$, which correspond to a iso $(1,2)$ algebra (the $2+1$ Poincaré algebra in the language of space-time motion groups). The associated skew-symmetric classical $r$-matrix is again the standard one [26] plus a pair of twists:

$$
\tilde{r}=r_{4}+r_{t 6}=-\frac{1}{2}\left(N_{+} \wedge J_{-}+J_{+} \wedge N_{-}\right)-\frac{1}{8} N_{3} \wedge J_{3}-\frac{1}{2} J_{+} \wedge J_{3} .
$$

We recall that previous works on twist deformations of $(3+1)$ Poincaré algebras were restricted to the study of abelian twists [29].

\subsection{Type $\left(\mathfrak{r}_{3}(1), \mathfrak{s}_{3}(0)\right)$}

Firstly note that this case is the $\omega \rightarrow 0$ limit of the type $\left(\mathfrak{r}_{3}(1), \mathfrak{s o}_{3} / \mathfrak{s l}_{2}\right)$, and thus it could be considered as a first step for the quantization of the latter. However, this DD algebra can be directly quantized and we get:

Coproduct:

$$
\begin{aligned}
& \Delta\left(x^{0}\right)=1 \otimes x^{0}+x^{0} \otimes 1 \\
& \Delta\left(x^{1}\right)=\cos \left(z \lambda x^{0}\right) \otimes x^{1}+\sin \left(z \lambda x^{0}\right) \otimes x^{2}+x^{1} \otimes \cos \left(z \lambda x^{0}\right)-x^{2} \otimes \sin \left(z \lambda x^{0}\right), \\
& \Delta\left(x^{2}\right)=-\sin \left(z \lambda x^{0}\right) \otimes x^{1}+\cos \left(z \lambda x^{0}\right) \otimes x^{2}+x^{1} \otimes \sin \left(z \lambda x^{0}\right)+x^{2} \otimes \cos \left(z \lambda x^{0}\right), \\
& \Delta\left(X_{0}\right)=1 \otimes X_{0}+X_{0} \otimes 1 \\
& \Delta\left(X_{1}\right)=e^{z X_{0}} \otimes X_{1}+X_{1} \otimes e^{-z X_{0}} \\
& \Delta\left(X_{2}\right)=e^{z X_{0}} \otimes X_{2}+X_{2} \otimes e^{-z X_{0}}
\end{aligned}
$$


Commutation rules:

a) Subalgebras:

$$
\begin{array}{lll}
{\left[x^{0}, x^{1}\right]=x^{1},} & {\left[X_{0}, X_{1}\right]=\lambda X_{2},} \\
{\left[x^{0}, x^{2}\right]=x^{2},} & {\left[X_{0}, X_{2}\right]=-\lambda X_{1},} \\
{\left[x^{1}, x^{2}\right]=0,} & {\left[X_{1}, X_{2}\right]=0 .}
\end{array}
$$

b) Crossed relations:

$$
\begin{array}{llll}
{\left[x^{0}, X_{0}\right]} & =0, & {\left[x^{1}, X_{0}\right]=-\lambda x^{2},} & {\left[x^{2}, X_{0}\right]=\lambda x^{1},} \\
{\left[x^{0}, X_{1}\right]=-X_{1},} & {\left[x^{1}, X_{1}\right]=\frac{\sinh \left(z X_{0}\right)}{z} \cos \left(z \lambda x^{0}\right),} & & {\left[x^{2}, X_{1}\right]=-\frac{\sin \left(z \lambda x^{0}\right)}{z} \cosh \left(z X_{0}\right),} \\
{\left[x^{0}, X_{2}\right]=-X_{2},} & {\left[x^{1}, X_{2}\right]=\frac{\sin \left(z \lambda x^{0}\right)}{z} \cosh \left(z X_{0}\right),} & & {\left[x^{2}, X_{2}\right]=\frac{\sinh \left(z X_{0}\right)}{z} \cos \left(z \lambda x^{0}\right) .}
\end{array}
$$

The relation with the deformations described in [26] can be easily derived, and this DD algebra $\mathfrak{s o}(1,3)$ has parameters $g_{(-\lambda,-1 / 2, \lambda)}$ and canonical $r$-matrix given by

$$
\tilde{r}=r_{s}+r_{t}=-\frac{1}{2}\left(N_{+} \wedge J_{-}+J_{+} \wedge N_{-}\right)-\frac{1}{8} N_{3} \wedge J_{3}
$$

Thus, we have again the standard deformation of $\mathfrak{s o}(1,3)$ plus a Reshetikhin twist $r_{t}$.

It is worthy to be emphasized that the canonical basis $\left\{x^{i}, X_{j}\right\}$ allows a more manageable description since the two Hopf subalgebras with classical commutation rules become apparent (compare (4) and (5) with the results in [26]).

\subsection{Type $\left(\mathfrak{r}_{3}(1), \mathfrak{n}_{3}\right)$}

This DD algebra is a contraction of both $\left(\mathfrak{r}_{3}(1), \mathfrak{s l}_{2}\right)$ and $\left(\mathfrak{r}_{3}(1), \mathfrak{r}_{3}(-1) / \mathfrak{s}_{3}(0)\right)$. Its quantization is simply the $\omega \rightarrow 0$ limit of the corresponding expressions in section 4.6.

Coproduct:

$$
\begin{array}{llrl}
\Delta\left(x^{0}\right)=1 \otimes x^{0}+x^{0} \otimes 1, & \Delta\left(X_{0}\right)=1 \otimes X_{0}+X_{0} \otimes 1, \\
\Delta\left(x^{1}\right)=\left(1 \otimes x^{1}+x^{1} \otimes 1\right)+z\left(x^{2} \otimes x^{0}-x^{2} \otimes x^{0}\right), & \Delta\left(X_{1}\right)=e^{z X_{0}} \otimes X_{1}+X_{1} \otimes e^{-z X_{0}}, \\
\Delta\left(x^{2}\right)=1 \otimes x^{2}+x^{2} \otimes 1, & \Delta\left(X_{2}\right)=e^{z X_{0}} \otimes X_{2}+X_{2} \otimes e^{-z X_{0}} .
\end{array}
$$

Commutation rules:

a) Subalgebras:

$$
\begin{array}{ll}
{\left[x^{0}, x^{1}\right]=x^{1},} & {\left[X_{0}, X_{1}\right]=0,} \\
{\left[x^{0}, x^{2}\right]=x^{2},} & {\left[X_{0}, X_{2}\right]=X_{1},} \\
{\left[x^{1}, x^{2}\right]=0,} & {\left[X_{1}, X_{2}\right]=0 .}
\end{array}
$$

b) Crossed relations:

$$
\begin{array}{llll}
{\left[x^{0}, X_{0}\right]=0,} & {\left[x^{1}, X_{0}\right]=x^{2},} & {\left[x^{2}, X_{0}\right]=0,} \\
{\left[x^{0}, X_{1}\right]=-X_{1},} & {\left[x^{1}, X_{1}\right]=\frac{\sinh \left(z X_{0}\right)}{z},} & {\left[x^{2}, X_{1}\right]=0,} \\
{\left[x^{0}, X_{2}\right]=-X_{2},} & {\left[x^{1}, X_{2}\right]=-x^{0} \cosh z X_{0},} & {\left[x^{2}, X_{2}\right]=\frac{\sinh \left(z X_{0}\right)}{z} .}
\end{array}
$$


Once again, we get the characterization $g_{(0,-1 / 2,1)}$. Not that by using the transformation $X_{1}{ }^{\prime}=-\lambda X_{1}, x^{1^{\prime}}=-\frac{1}{\lambda} x^{1}$, the type $\left(\mathfrak{r}_{3}(1), \mathfrak{n}_{3}\right)$ is just the limit for $\lambda \rightarrow 0$ of the known type $\left(\mathfrak{r}_{3}(1), \mathfrak{s}_{3}(0)\right)$. The associated skew-symmetric classical $r$-matrix is:

$$
\tilde{r}=r_{s}+r_{t}=-\frac{1}{2}\left(N_{+} \wedge J_{-}+J_{+} \wedge N_{-}\right)-\frac{1}{8} N_{3} \wedge J_{3}
$$

Thus, we have again the Poincaré analogue to the previous case: the known standard deformation [26] plus a twist.

\subsection{Types $\left(\mathfrak{r}_{3}(1), \mathfrak{r}_{3}(-1) / \mathfrak{s}_{3}(0)\right)$}

We remark that we have $\left(\mathfrak{r}_{3}(1), \mathfrak{r}_{3}(-1)\right)$ for $\omega=+1$, and $\left(\mathfrak{r}_{3}(1), \mathfrak{s}_{3}(0)\right)$ for $\omega=-1$. The full quantization is as follows.

Coproduct:

$$
\begin{aligned}
& \Delta\left(x^{0}\right)=\cosh \left(\sqrt{\omega} z x^{2}\right) \otimes x^{0}+\sqrt{\omega} \sinh \left(\sqrt{\omega} z x^{2}\right) \otimes x^{1} \\
& +x^{0} \otimes \cosh \left(\sqrt{\omega} z x^{2}\right)-\sqrt{\omega} x^{1} \otimes \sinh \left(\sqrt{\omega} z x^{2}\right), \\
& \Delta\left(x^{1}\right)=\frac{\sinh \left(\sqrt{\omega} z x^{2}\right)}{\sqrt{\omega}} \otimes x^{0}+\cosh \left(\sqrt{\omega} z x^{2}\right) \otimes x^{1} \\
& -x^{0} \otimes \frac{\sinh \left(\sqrt{\omega} z x^{2}\right)}{\sqrt{\omega}}+x^{1} \otimes \cosh \left(\sqrt{\omega} z x^{2}\right), \\
& \Delta\left(x^{2}\right)=1 \otimes x^{2}+x^{2} \otimes 1, \\
& \Delta\left(X_{0}\right)=1 \otimes X_{0}+X_{0} \otimes 1 \text {, } \\
& \Delta\left(X_{1}\right)=e^{z X_{0}} \otimes X_{1}+X_{1} \otimes e^{-z X_{0}}, \\
& \Delta\left(X_{2}\right)=e^{z X_{0}} \otimes X_{2}+X_{2} \otimes e^{-z X_{0}} .
\end{aligned}
$$

Commutation rules:

a) Subalgebras:

$$
\begin{array}{lll}
{\left[x^{0}, x^{1}\right]=x^{1} \cosh \left[\sqrt{\omega} z x^{2}\right],} & {\left[X_{0}, X_{1}\right]=0,} \\
{\left[x^{0}, x^{2}\right]=\frac{\sinh \left[\sqrt{\omega} z x^{2}\right]}{\sqrt{\omega} z},} & {\left[X_{0}, X_{2}\right]=X_{1},} \\
{\left[x^{1}, x^{2}\right]=0,} & {\left[X_{1}, X_{2}\right]=\omega \frac{\sinh \left(2 z X_{0}\right)}{2 z} .}
\end{array}
$$

b) Crossed relations:

$$
\begin{array}{rlrl}
{\left[x^{0}, X_{0}\right]} & =0, & \\
{\left[x^{0}, X_{1}\right]=\frac{\sqrt{\omega}}{z} \sinh \left[\sqrt{\omega} z x^{2}\right] \cosh \left[z X_{0}\right]-\cosh \left[\sqrt{\omega} z x^{2}\right] X_{1},} & \\
{\left[x^{0}, X_{2}\right]=-\omega x^{1} \cosh \left(z X_{0}\right)-X_{2} \cosh \left(\sqrt{\omega} z x^{2}\right),} & \\
{\left[x^{1}, X_{0}\right]=\frac{\sinh \left[\sqrt{\omega} z x^{2}\right]}{\sqrt{\omega} z},} & {\left[x^{2}, X_{0}\right]=0,} \\
{\left[x^{1}, X_{1}\right]=\cosh \left[\sqrt{\omega} z x^{2}\right] \frac{\sinh \left[z X_{0}\right]}{z},} & {\left[x_{1}^{2}, X_{2}\right]=0, \frac{\sinh \left(z X_{0}\right)}{z} .} \\
{\left[x^{1}, X_{2}\right]=-x^{0} \cosh \left(z X_{0}\right),} &
\end{array}
$$


Note that the use of a fully symmetric basis is implicit since

$$
\begin{aligned}
{\left[x^{0}, X_{2}\right] } & =-\omega x^{1} \cosh \left(z X_{0}\right)-X_{2} \cosh \left(\sqrt{\omega} z x^{2}\right) \\
& =-\omega \operatorname{Sym}\left[x^{1} \cosh \left(z X_{0}\right)\right]-\operatorname{Sym}\left[X_{2} \cosh \left(\sqrt{\omega} z x^{2}\right)\right],
\end{aligned}
$$

where Sym is a linear operator such that

$$
\operatorname{Sym}\left\{A_{1} \ldots A_{n}\right\}:=\frac{1}{n !} \sum_{p \in \mathrm{S}_{\mathrm{n}}} p\left(A_{1} \ldots A_{n}\right),
$$

with $\mathrm{S}_{\mathrm{n}}$ the permutation group of $n$ elements (see [25]).

In this case the DD algebra is a $i s o(2,1)$ algebra with parameters $g_{(1,-1 / 2,0)}$ and the classical $r$-matrix would be

$$
\tilde{r}=r_{s}+r_{t 1}+r_{t 2}=-\frac{1}{2}\left(N_{+} \wedge J_{-}+J_{+} \wedge N_{-}\right)-\frac{1}{8} N_{3} \wedge J_{3}-\frac{\omega}{2} N_{+} \wedge J_{+} .
$$

Once again, we have the standard deformation of the $2+1$ Poincaré algebra plus two noncommuting twists, with the same interpretation as for the type $\left(\mathfrak{r}_{3}(1), \mathfrak{s l}_{2}\right)$ for $\mathfrak{s o}(1,3)$.

\section{$5 \quad$ Quantum DD algebras: $\mathfrak{g}=\mathfrak{r}_{3}(\rho)$}

The second set of quantum DD algebras corresponds to the quantization of those DD algebras with $\mathfrak{g}=\mathfrak{r}_{3}(\rho)$. The three possible cases are described in Table III and can be fully quantized.

Table II. DD algebras with $\mathfrak{g}=\mathfrak{r}_{3}(\rho)$.

\begin{tabular}{|c|c|c|c|}
\hline$\left(\mathfrak{g}, \mathfrak{g}^{*}\right)$ & $\left(\mathfrak{r}_{3}(\rho), \mathfrak{n}_{3}\right)$ & $\left(\mathfrak{r}_{3}(\rho), \mathfrak{r}_{3}(-\rho)\right)$ & $\left(\mathfrak{r}_{3}(\rho), \mathfrak{r}_{3}(-\rho)\right)$ \\
\hline $\mathrm{DD}$ & $\mathfrak{r}_{3}(\rho) \odot R^{3}$ & $\mathfrak{r}_{3}(\rho) \odot R^{3}$ & $s l_{2} \oplus s l_{2}$ \\
\hline$[23] \mathrm{Nr}$. & 5 & 6 & 7 \\
\hline \hline$\left[x^{0}, x^{1}\right]$ & $x^{1}$ & $x^{1}$ & $x^{1}$ \\
\hline$\left[x^{0}, x^{2}\right]$ & $\rho x^{2}$ & $\rho x^{2}$ & $\rho x^{2}$ \\
\hline$\left[x^{1}, x^{2}\right]$ & 0 & 0 & 0 \\
\hline$\left[X_{0}, X_{1}\right]$ & 0 & $X_{0}$ & $\lambda X_{1}$ \\
\hline$\left[X_{0}, X_{2}\right]$ & 0 & 0 & $-\lambda \rho X_{2}$ \\
\hline$\left[X_{1}, X_{2}\right]$ & $(1+\rho) X_{0}$ & $\rho X_{2}$ & 0 \\
\hline$\left[x^{0}, X_{0}\right]$ & 0 & $x^{1}$ & 0 \\
\hline$\left[x^{0}, X_{1}\right]$ & $(1+\rho) x^{2}-X_{1}$ & $-x^{0}-X_{1}$ & $-X_{1}$ \\
\hline$\left[x^{0}, X_{2}\right]$ & $-(1+\rho) x^{1}-\rho X_{2}$ & $-\rho X_{2}$ & $-\rho X_{2}$ \\
\hline$\left[x^{1}, X_{0}\right]$ & 0 & 0 & $\lambda x^{1}$ \\
\hline$\left[x^{1}, X_{1}\right]$ & $X_{0}$ & $X_{0}$ & $-\lambda x^{0}+X_{0}$ \\
\hline$\left[x^{1}, X_{2}\right]$ & 0 & 0 & 0 \\
\hline$\left[x^{2}, X_{0}\right]$ & 0 & 0 & $-\lambda \rho x^{2}$ \\
\hline$\left[x^{2}, X_{1}\right]$ & 0 & $\rho x^{2}$ & 0 \\
\hline$\left[x^{2}, X_{2}\right]$ & $\rho X_{0}$ & $\rho\left(-x^{1}+X_{0}\right)$ & $\rho\left(\lambda x^{0}+X_{0}\right)$ \\
\hline
\end{tabular}




\subsection{Case $\left(\mathfrak{r}_{3}(\rho), \mathfrak{n}_{3}\right)$}

Coproduct:

$$
\begin{aligned}
& \Delta\left(x^{0}\right)=1 \otimes x^{0}+x^{0} \otimes 1-z(1+\rho)\left(x^{1} \otimes x^{2}-x^{2} \otimes x^{1}\right) \\
& \Delta\left(X_{0}\right)=1 \otimes X_{0}+X_{0} \otimes 1 \\
& \Delta\left(x^{1}\right)=1 \otimes x^{1}+x^{1} \otimes 1 \\
& \Delta\left(X_{1}\right)=e^{z X_{0}} \otimes X_{1}+X_{1} \otimes e^{-z X_{0}} \\
& \Delta\left(x^{2}\right)=1 \otimes x^{2}+x^{2} \otimes 1 \\
& \Delta\left(X_{2}\right)=e^{z \rho X_{0}} \otimes X_{2}+X_{2} \otimes e^{-z \rho X_{0}}
\end{aligned}
$$

Commutation rules:

a) Subalgebras:

$$
\begin{array}{ll}
{\left[x^{0}, x^{1}\right]=x^{1},} & {\left[X_{0}, X_{1}\right]=0,} \\
{\left[x^{0}, x^{2}\right]=\rho x^{2},} & {\left[X_{0}, X_{2}\right]=0,} \\
{\left[x^{1}, x^{2}\right]=0,} & {\left[X_{1}, X_{2}\right]=\frac{\sinh \left(z(1+\rho) X_{0}\right)}{z} .}
\end{array}
$$

b) Crossed relations:

$$
\begin{array}{llll}
{\left[x^{0}, X_{0}\right]} & =0, & {\left[x^{1}, X_{0}\right]=0,} & {\left[x^{2}, X_{0}\right]=0,} \\
{\left[x^{0}, X_{1}\right]=(1+\rho) x^{2} \cosh \left(z X_{0}\right)-X_{1},} & {\left[x^{1}, X_{1}\right]=\frac{\sinh \left(z X_{0}\right)}{z},} & {\left[x^{2}, X_{1}\right]=0,} \\
{\left[x^{0}, X_{2}\right]=-(1+\rho) x^{1} \cosh \left(z \rho X_{0}\right)-\rho X_{2},} & {\left[x^{1}, X_{2}\right]=0,} & {\left[x^{2}, X_{2}\right]=\frac{\sinh \left(z \rho X_{0}\right)}{z} .}
\end{array}
$$

\subsection{Case $\left(\mathfrak{r}_{3}(\rho), \mathfrak{r}_{3}(-\rho)\right)$}

Coproduct:

$$
\begin{array}{ll}
\Delta\left(x^{0}\right)=e^{z x^{1}} \otimes x^{0}+x^{0} \otimes e^{-z x^{1}}, & \Delta\left(X_{0}\right)=1 \otimes X_{0}+X_{0} \otimes 1, \\
\Delta\left(x^{1}\right)=1 \otimes x^{1}+x^{1} \otimes 1, & \Delta\left(X_{1}\right)=e^{z X_{0}} \otimes X_{1}+X_{1} \otimes e^{-z X_{0}}, \\
\Delta\left(x^{2}\right)=e^{-z \rho x^{1}} \otimes x^{2}+x^{2} \otimes e^{z \rho x^{1}}, & \Delta\left(X_{2}\right)=e^{z \rho X_{0}} \otimes X_{2}+X_{2} \otimes e^{-z \rho X_{0}} .
\end{array}
$$

Commutation rules:

a) Subalgebras:

$$
\begin{array}{llrl}
{\left[x^{0}, x^{1}\right]} & =\frac{\sinh \left(z x^{1}\right)}{z}, & & {\left[X_{0}, X_{1}\right]=\frac{\sinh \left(z X_{0}\right)}{z},} \\
{\left[x^{0}, x^{2}\right]=\rho x^{2} \cosh \left(z x^{1}\right),} & {\left[X_{0}, X_{2}\right]=0,} \\
{\left[x^{1}, x^{2}\right]=0,} & {\left[X_{1}, X_{2}\right]=\rho X_{2} \cosh \left(z X_{0}\right) .}
\end{array}
$$


b) Crossed relations:

$$
\begin{aligned}
{\left[x^{0}, X_{0}\right]=} & \frac{\sinh \left(z x^{1}\right)}{z}, & {\left[x^{1}, X_{0}\right]=0, } & {\left[x^{2}, X_{0}\right]=0, } \\
{\left[x^{0}, X_{1}\right]=-\cosh \left(z X_{0}\right) x^{0} } & {\left[x^{1}, X_{1}\right]=\frac{\sinh \left(z X_{0}\right)}{z}, } & & {\left[x^{2}, X_{1}\right]=\rho x^{2} \cosh \left(z X_{0}\right), } \\
& -\cosh \left(z x^{1}\right) X_{1}, & & \\
{\left[x^{0}, X_{2}\right]=-\rho X_{2} \cosh \left(z x^{1}\right), } & {\left[x^{1}, X_{2}\right]=0, } & & {\left[x^{2}, X_{2}\right]=-\frac{\sinh \left(z \rho\left(-x^{1}+X_{0}\right)\right)}{z} . }
\end{aligned}
$$

The symmetrization prescription is again preserved despite the non-symmetric shape of some brackets. Note that this DD algebra is self-dual for $\rho=0$.

\subsection{Case $\left(\mathfrak{r}_{3}(\rho), \mathfrak{r}_{3}(-\rho)\right)$}

Coproduct:

$$
\begin{array}{ll}
\Delta\left(x^{0}\right)=1 \otimes x^{0}+x^{0} \otimes 1, & \Delta\left(X_{0}\right)=1 \otimes X_{0}+X_{0} \otimes 1, \\
\Delta\left(x^{1}\right)=e^{-z \lambda x^{0}} \otimes x^{1}+x^{1} \otimes e^{z \lambda x^{0}}, & \Delta\left(X_{1}\right)=e^{z X_{0}} \otimes X_{1}+X_{1} \otimes e^{-z X_{0}}, \\
\Delta\left(x^{2}\right)=e^{z \lambda \rho x^{0}} \otimes x^{2}+x^{2} \otimes e^{-z \lambda \rho x^{0}}, & \Delta\left(X_{2}\right)=e^{z \rho X_{0}} \otimes X_{2}+X_{2} \otimes e^{-z \rho X_{0}} .
\end{array}
$$

Commutation rules:

a) Subalgebras:

$$
\begin{array}{ll}
{\left[x^{0}, x^{1}\right]=x^{1},} & {\left[X_{0}, X_{1}\right]=\lambda X_{1},} \\
{\left[x^{0}, x^{2}\right]=\rho x^{2},} & {\left[X_{0}, X_{2}\right]=-\lambda \rho X_{2},} \\
{\left[x^{1}, x^{2}\right]=0,} & {\left[X_{1}, X_{2}\right]=0}
\end{array}
$$

b) Crossed relations:

$$
\begin{array}{llll}
{\left[x^{0}, X_{0}\right]=0,} & {\left[x^{1}, X_{0}\right]=\lambda x^{1},} & {\left[x^{2}, X_{0}\right]=-\lambda \rho x^{2},} \\
{\left[x^{0}, X_{1}\right]=-X_{1},} & {\left[x^{1}, X_{1}\right]=\frac{\sinh \left(z\left(-\lambda x^{0}+X_{0}\right)\right)}{z},} & {\left[x^{2}, X_{1}\right]=0,} \\
{\left[x^{0}, X_{2}\right]=-\rho X_{2},} & {\left[x^{1}, X_{2}\right]=0,} & {\left[x^{2}, X_{2}\right]=\frac{\sinh \left(z \rho\left(\lambda x^{0}+X_{0}\right)\right)}{z} .}
\end{array}
$$

Note that the quantum DD algebra corresponding to $g l(2)$ is included as a subalgebra in several different ways. Again, this DD algebra is self-dual for $\rho=0$.

\section{Quantum DD algebras: $\mathfrak{g}=\left\{\mathfrak{r}_{3}(-1), \mathfrak{r}_{3}^{\prime}(1)\right\}$}

The following set of quantum DD algebras (see Table IV below) can be also completely quantized by using a direct quantization approach. Note that all the DD algebras have a semidirect product structure. 
Table IV. DD algebras with $\mathfrak{g}=\left\{\mathfrak{r}_{3}(-1), \mathfrak{r}_{3}^{\prime}(1)\right\}$.

\begin{tabular}{|c|c|c|c|c|c|}
\hline$\left(\mathfrak{g}, \mathfrak{g}^{*}\right)$ & $\left(\mathfrak{r}_{3}(-1), \mathfrak{n}_{3}\right)$ & $\left(\mathfrak{r}_{3}(-1), \mathfrak{r}_{3}^{\prime}(1)\right)$ & $\left(\mathfrak{r}_{3}(-1), \mathfrak{r}_{3}^{\prime}(1)\right)$ & $\left(\mathfrak{r}_{3}^{\prime}(1), \mathfrak{n}_{3}\right)$ & $\left(\mathfrak{r}_{3}^{\prime}(1), \mathfrak{n}_{3}\right)$ \\
\hline $\mathrm{DD}$ & $\mathfrak{r}_{3}^{\prime}(1) \odot R^{3}$ & $\mathfrak{r}_{3}^{\prime}(1) \odot R^{3}$ & $\mathfrak{s l}_{2} \odot R^{3}$ & $\mathfrak{r}_{3}^{\prime}(1) \odot R^{3}$ & $s l_{2} \odot R^{3}$ \\
\hline$[23, \mathrm{Nr}$. & $5^{\prime}$ & 8 & $(14)$ & 12 & 13 \\
\hline \hline$\left[x^{0}, x^{1}\right]$ & $x^{1}$ & $x^{1}$ & $x^{1}$ & $x^{1}$ & $x^{1}$ \\
\hline$\left[x^{0}, x^{2}\right]$ & $-x^{2}$ & $-x^{2}$ & $-x^{2}$ & $x^{1}+x^{2}$ & $x^{1}+x^{2}$ \\
\hline$\left[x^{1}, x^{2}\right]$ & 0 & 0 & 0 & 0 & 0 \\
\hline$\left[X_{0}, X_{1}\right]$ & 0 & $X_{0}$ & $X_{0}$ & 0 & $\lambda X_{2}$ \\
\hline$\left[X_{0}, X_{2}\right]$ & 0 & 0 & $-\lambda X_{0}$ & 0 & 0 \\
\hline$\left[X_{1}, X_{2}\right]$ & $X_{0}$ & $X_{0}-X_{2}$ & $X_{0}-\lambda X_{1}-X_{2}$ & $\omega X_{0}$ & 0 \\
\hline$\left[x^{0}, X_{0}\right]$ & 0 & $x^{1}$ & $x^{1}-\lambda x^{2}$ & 0 & 0 \\
\hline$\left[x^{0}, X_{1}\right]$ & $x^{2}-X_{1}$ & $-X_{1}-x^{0}+x^{2}$ & $-X_{1}-x^{0}+x^{2}$ & $\omega x^{2}-X_{1}-X_{2}$ & $-X_{1}-X_{2}$ \\
\hline$\left[x^{0}, X_{2}\right]$ & $-x^{1}+X_{2}$ & $-x^{1}+X_{2}$ & $\lambda x^{0}-x^{1}+X_{2}$ & $-\omega x^{1}-X_{2}$ & $-X_{2}$ \\
\hline$\left[x^{1}, X_{0}\right]$ & 0 & 0 & 0 & 0 & 0 \\
\hline$\left[x^{1}, X_{1}\right]$ & $X_{0}$ & $X_{0}$ & $-\lambda x^{2}+X_{0}$ & $X_{0}$ & $X_{0}$ \\
\hline$\left[x^{1}, X_{2}\right]$ & 0 & 0 & $\lambda x^{1}$ & 0 & 0 \\
\hline$\left[x^{2}, X_{0}\right]$ & 0 & 0 & 0 & 0 & $\lambda x^{1}$ \\
\hline$\left[x^{2}, X_{1}\right]$ & 0 & $-x^{2}$ & $-x^{2}$ & $X_{0}$ & $X_{0}-\lambda x^{0}$ \\
\hline$\left[x^{2}, X_{2}\right]$ & $-X_{0}$ & $x^{1}-X_{0}$ & $x^{1}-X_{0}$ & $X_{0}$ & $X_{0}$ \\
\hline
\end{tabular}

\subsection{Case $\left(\mathfrak{r}_{3}(-1), \mathfrak{n}_{3}\right)$}

\section{Coproduct:}

$$
\begin{array}{llrl}
\Delta\left(x^{0}\right) & =1 \otimes x^{0}+x^{0} \otimes 1-z\left(x^{1} \otimes x^{2}-x^{2} \otimes x^{1}\right), & & \Delta\left(X_{0}\right)=1 \otimes X_{0}+X_{0} \otimes 1, \\
\Delta\left(x^{1}\right) & =1 \otimes x^{1}+x^{1} \otimes 1, & \Delta\left(X_{1}\right) & =e^{z X_{0}} \otimes X_{1}+X_{1} \otimes e^{-z X_{0}}, \\
\Delta\left(x^{2}\right) & =1 \otimes x^{2}+x^{2} \otimes 1, & \Delta\left(X_{2}\right) & =e^{-z X_{0}} \otimes X_{2}+X_{2} \otimes e^{z X_{0}} .
\end{array}
$$

Commutation rules:

a) Subalgebras:

$$
\begin{array}{ll}
{\left[x^{0}, x^{1}\right]=x^{1},} & {\left[X_{0}, X_{1}\right]=0,} \\
{\left[x^{0}, x^{2}\right]=-x^{2},} & {\left[X_{0}, X_{2}\right]=0,} \\
{\left[x^{1}, x^{2}\right]=0,} & {\left[X_{1}, X_{2}\right]=X_{0} .}
\end{array}
$$

b) Crossed relations:

$$
\begin{array}{llrl}
{\left[x^{0}, X_{0}\right]} & =0, & {\left[x^{1}, X_{0}\right]=0,} & {\left[x^{2}, X_{0}\right]=0,} \\
{\left[x^{0}, X_{1}\right]=x^{2} \cosh \left(z X_{0}\right)-X_{1},} & {\left[x^{1}, X_{1}\right]=\frac{\sinh \left(z X_{0}\right)}{z},} & {\left[x^{2}, X_{1}\right]=0,} \\
{\left[x^{0}, X_{2}\right]=-x^{1} \cosh \left(z X_{0}\right)+X_{2},} & {\left[x^{1}, X_{2}\right]=0,} & {\left[x^{2}, X_{2}\right]=-\frac{\sinh \left(z X_{0}\right)}{z} .}
\end{array}
$$




\subsection{Case $\left(\mathfrak{r}_{3}(-1), \mathfrak{r}_{3}^{\prime}(1)\right)$}

Coproduct:

$$
\begin{aligned}
& \Delta\left(x^{0}\right)=e^{z x^{1}} \otimes x^{0}+x^{0} \otimes e^{-z x^{1}}-z\left(x^{1} e^{z x^{1}} \otimes x^{2}-x^{2} \otimes x^{1} e^{-z x^{1}}\right), \\
& \Delta\left(x^{1}\right)=1 \otimes x^{1}+x^{1} \otimes 1 \\
& \Delta\left(x^{2}\right)=e^{z x^{1}} \otimes x^{2}+x^{2} \otimes e^{-z x^{1}} \\
& \Delta\left(X_{0}\right)=1 \otimes X_{0}+X_{0} \otimes 1 \\
& \Delta\left(X_{1}\right)=e^{z X_{0}} \otimes X_{1}+X_{1} \otimes e^{-z X_{0}} \\
& \Delta\left(X_{2}\right)=e^{-z X_{0}} \otimes X_{2}+X_{2} \otimes e^{z X_{0}} .
\end{aligned}
$$

Commutation rules:

a) Subalgebras:

$$
\begin{array}{rlrl}
{\left[x^{0}, x^{1}\right]} & =\frac{\sinh \left(z x^{1}\right)}{z}, & & {\left[X_{0}, X_{1}\right]=\frac{\sinh \left(z X_{0}\right)}{z},} \\
{\left[x^{0}, x^{2}\right]} & =-x^{2} \cosh \left(z x^{1}\right), & & {\left[X_{0}, X_{2}\right]=0,} \\
{\left[x^{1}, x^{2}\right]} & =0, & {\left[X_{1}, X_{2}\right]=X_{0}-X_{2} \cosh \left(z X_{0}\right) .}
\end{array}
$$

b) Crossed relations:

$$
\begin{aligned}
{\left[x^{0}, X_{0}\right] } & =\frac{\sinh \left(z x^{1}\right)}{z}, & & \\
{\left[x^{0}, X_{1}\right] } & =-X_{1} \cosh \left(z x^{1}\right)-x^{0} \cosh \left(z X_{0}\right)+x^{2} \cosh \left(z X_{0}\right) & & \\
{\left[x^{0}, X_{2}\right] } & =-x^{1} \cosh \left(z\left(x^{1}-X_{0}\right)\right)+X_{2} \cosh \left(z x^{1}\right), & & \\
{\left[x^{1}, X_{0}\right] } & =0, & & {\left[x^{2}, X_{0}\right]=0 } \\
{\left[x^{1}, X_{1}\right] } & =\frac{\sinh \left(z X_{0}\right)}{z}, & {\left[x^{2}, X_{1}\right] } & =-x^{2} \cosh \left(z X_{0}\right), \\
{\left[x^{1}, X_{2}\right] } & =0, & &
\end{aligned}
$$

\subsection{Case $\left(\mathfrak{r}_{3}(-1), \mathfrak{r}_{3}^{\prime}(1)\right)$}

In order to get the explicit quantization, the following pairing-preserving change of basis turns out to be convenient:

$$
\begin{array}{ll}
Y_{0}=X_{0}, & y^{0}=x^{0}, \\
Y_{1}=\frac{1}{\sqrt{1+\lambda^{2}}}\left(\lambda X_{1}+X_{2}\right), & y^{1}=\frac{1}{\sqrt{1+\lambda^{2}}}\left(\lambda x^{1}+x^{2}\right), \\
Y_{2}=\frac{1}{\sqrt{1+\lambda^{2}}}\left(X_{1}-\lambda X_{2}\right), & y^{2}=\frac{1}{\sqrt{1+\lambda^{2}}}\left(x^{1}-\lambda x^{2}\right) .
\end{array}
$$

In this new basis, the quantum DD algebra reads: 
Coproduct:

$$
\begin{aligned}
& \Delta\left(y^{0}\right)=e^{-z \sqrt{1+\lambda^{2}} y^{2}} \otimes y^{0}+y^{0} \otimes e^{z \sqrt{1+\lambda^{2}} y^{2}}-z\left(y^{1} \otimes y^{2} e^{z \sqrt{1+\lambda^{2}} y^{2}}-y^{2} e^{-z \sqrt{1+\lambda^{2}} y^{2}} \otimes y^{1}\right) \\
& \Delta\left(y^{1}\right)=e^{-z \sqrt{1+\lambda^{2}} y^{2}} \otimes y^{1}+y^{1} \otimes e^{z \sqrt{1+\lambda^{2}} y^{2}}, \\
& \Delta\left(y^{2}\right)=1 \otimes y^{2}+y^{2} \otimes 1 \\
& \Delta\left(Y_{0}\right)=1 \otimes Y_{0}+Y_{0} \otimes 1, v \Delta\left(Y_{1}\right)=\frac{1}{1+\lambda^{2}}\left\{\left(e^{z Y_{0}}+\lambda^{2} e^{-z Y_{0}}\right) \otimes Y_{1}+Y_{1} \otimes\left(e^{-z Y_{0}}+\lambda^{2} e^{z Y_{0}}\right) v\right. \\
& \left.-2 \lambda \sinh \left(z Y_{0}\right) \otimes Y_{2}+2 \lambda Y_{2} \otimes \sinh \left(z Y_{0}\right)\right\} \\
& \Delta\left(Y_{2}\right)=\frac{1}{1+\lambda^{2}}\left\{\left(e^{-z Y_{0}}+\lambda^{2} e^{z Y_{0}}\right) \otimes Y_{2}+Y_{2} \otimes\left(e^{z Y_{0}}+\lambda^{2} e^{-z Y_{0}}\right)\right. \\
& \left.-2 \lambda \sinh \left(z Y_{0}\right) \otimes Y_{1}+2 \lambda Y_{1} \otimes \sinh \left(z Y_{0}\right)\right\} \text {. }
\end{aligned}
$$

\section{Commutation rules:}

a) Subalgebras:

$$
\begin{aligned}
& {\left[y^{0}, y^{1}\right]=-\frac{1-\lambda^{2}}{1+\lambda^{2}} y^{1} \cosh \left(z \sqrt{1+\lambda^{2}} y^{2}\right)+\frac{2 \lambda}{1+\lambda^{2}} \frac{\sinh \left(2 z \sqrt{1+\lambda^{2}} y^{2}\right)}{2 z \sqrt{1+\lambda^{2}}}} \\
& {\left[y^{0}, y^{2}\right]=\frac{2 \lambda}{1+\lambda^{2}} y^{1}+\frac{1-\lambda^{2}}{1+\lambda^{2}} \frac{\sinh \left(z \sqrt{1+\lambda^{2}} y^{2}\right)}{z \sqrt{1+\lambda^{2}}}} \\
& {\left[y^{1}, y^{2}\right]=0} \\
& {\left[Y_{0}, Y_{1}\right]=0} \\
& {\left[Y_{0}, Y_{2}\right]=\sqrt{1+\lambda^{2}} \frac{\sinh \left(z Y_{0}\right)}{z}} \\
& {\left[Y_{1}, Y_{2}\right]=-Y_{0}+\sqrt{1+\lambda^{2}} Y_{1} \cosh \left(z Y_{0}\right)}
\end{aligned}
$$

b) Crossed relations:

$$
\begin{aligned}
{\left[y^{0}, Y_{0}\right]=} & \frac{\sinh \left(z \sqrt{1+\lambda^{2}} y^{2}\right)}{z} \\
{\left[y^{0}, Y_{1}\right]=} & \left.\frac{1-\lambda^{2}}{1+\lambda^{2}} Y_{1}-\frac{2 \lambda}{1+\lambda^{2}} Y_{2}\right) \cosh \left(z \sqrt{1+\lambda^{2}} y^{2}\right) \\
& -y^{2} \cosh \left(z \sqrt{1+\lambda^{2}} y^{2}\right)+\frac{1-\lambda^{2}}{1+\lambda^{2}} y^{2} \sinh \left(z \sqrt{1+\lambda^{2}} y^{2}\right) \sinh \left(z Y_{0}\right), \\
{\left[y^{0}, Y_{2}\right]=- } & \sqrt{1+\lambda^{2}} y^{0} \cosh \left(z Y_{0}\right)-\left(\frac{2 \lambda}{1+\lambda^{2}} Y_{1}-\frac{1-\lambda^{2}}{1+\lambda^{2}} Y_{2}\right) \cosh \left(z \sqrt{1+\lambda^{2}} y^{2}\right) \\
& -\frac{2 \lambda}{1+\lambda^{2}} y^{2} \sinh \left(z \sqrt{1+\lambda^{2}} y^{2}\right)+y^{1} \cosh \left(z Y_{0}\right) \\
{\left[y^{1}, Y_{0}\right]=} & 0, \quad \\
{\left[y^{1}, Y_{1}\right]=} & \frac{\sinh \left(z \sqrt{1+\lambda^{2}} y^{2}\right)}{z} \cosh \left(z Y_{0}\right)-\frac{1-\lambda^{2}}{1+\lambda^{2}} \frac{\sinh \left(z Y_{0}\right)}{z} \cosh \left(z \sqrt{1+\lambda^{2}} y^{2}\right) \\
{\left[y^{1}, Y_{2}\right]=} & -\sqrt{1+\lambda^{2}} y^{1} \cosh \left(z Y_{0}\right)+\frac{2 \lambda}{1+\lambda^{2}} \frac{\sinh \left(z Y_{0}\right)}{z} \cosh \left(z \sqrt{1+\lambda^{2}} y^{2}\right) \\
{\left[y^{2}, Y_{1}\right]=} & \frac{2 \lambda}{1+\lambda^{2}} \frac{\sinh \left(z Y_{0}\right)}{z}, \\
{\left[y^{2}, Y_{2}\right]=} & \frac{1-\lambda^{2}}{1+\lambda^{2}} \frac{\sinh \left(z Y_{0}\right)}{z} .
\end{aligned}
$$




\subsection{Case $\left(\mathfrak{r}_{3}^{\prime}(1), \mathfrak{n}_{3}\right)$}

Coproduct:

$$
\begin{aligned}
& \Delta\left(x^{0}\right)=1 \otimes x^{0}+x^{0} \otimes 1+\omega z\left(x^{1} \otimes x^{2}-x^{2} \otimes x^{1}\right), \\
& \Delta\left(x^{1}\right)=1 \otimes x^{1}+x^{1} \otimes 1 \\
& \Delta\left(x^{2}\right)=1 \otimes x^{2}+x^{2} \otimes 1 \\
& \Delta\left(X_{0}\right)=1 \otimes X_{0}+X_{0} \otimes 1 \\
& \Delta\left(X_{1}\right)=e^{-z X_{0}} \otimes X_{1}+X_{1} \otimes e^{z X_{0}}-z\left(X_{0} e^{-z X_{0}} \otimes X_{2}-X_{2} \otimes X_{0} e^{z X_{0}}\right), \\
& \Delta\left(X_{2}\right)=e^{-z X_{0}} \otimes X_{2}+X_{2} \otimes e^{z X_{0}},
\end{aligned}
$$

Commutation rules:

a) Subalgebras:

$$
\begin{array}{llrl}
{\left[x^{0}, x^{1}\right]} & =x^{1}, & {\left[X_{0}, X_{1}\right]=0,} \\
{\left[x^{0}, x^{2}\right]=x^{1}+x^{2},} & {\left[X_{0}, X_{2}\right]=0,} \\
{\left[x^{1}, x^{2}\right]=0,} & {\left[X_{1}, X_{2}\right]=\omega \frac{\sinh \left(2 z X_{0}\right)}{2 z} .}
\end{array}
$$

b) Crossed relations:

$$
\begin{array}{rlrl}
{\left[x^{0}, X_{0}\right]} & =0, & \\
{\left[x^{0}, X_{1}\right]} & =-X_{1}-X_{2}+\omega x^{2} \cosh \left(z X_{0}\right) & & \\
& \quad-\omega z X_{0} x^{1} \sinh \left(z X_{0}\right), & & \\
{\left[x^{0}, X_{2}\right]} & =-X_{2}-\omega x^{1} \cosh \left(z X_{0}\right), & & \\
{\left[x^{1}, X_{0}\right]} & =0, & & {\left[x^{2}, X_{0}\right]=0,} \\
{\left[x^{1}, X_{1}\right]} & =\frac{\sinh \left(z X_{0}\right)}{z}, & & {\left[x^{2}\right]=\frac{\sinh \left(z X_{0}\right)}{z} .}
\end{array}
$$

\subsection{Case $\left(\mathfrak{r}_{3}^{\prime}(1), \mathfrak{n}_{3}\right)$}

Coproduct:

$$
\begin{aligned}
& \Delta\left(x^{0}\right)=1 \otimes x^{0}+x^{0} \otimes 1, \\
& \Delta\left(x^{1}\right)=1 \otimes x^{1}+x^{1} \otimes 1, \\
& \Delta\left(x^{2}\right)=1 \otimes x^{2}+x^{2} \otimes 1+\lambda z\left(x^{0} \otimes x^{1}-x^{1} \otimes x^{0}\right), \\
& \Delta\left(X_{0}\right)=1 \otimes X_{0}+X_{0} \otimes 1, \\
& \Delta\left(X_{1}\right)=e^{-z X_{0}} \otimes X_{1}+X_{1} \otimes e^{z X_{0}}-z\left(X_{0} e^{-z X_{0}} \otimes X_{2}-X_{2} \otimes X_{0} e^{z X_{0}}\right), \\
& \Delta\left(X_{2}\right)=e^{-z X_{0}} \otimes X_{2}+X_{2} \otimes e^{z X_{0}} .
\end{aligned}
$$

Commutation rules: 
a) Subalgebras:

$$
\begin{array}{llrl}
{\left[x^{0}, x^{1}\right]} & =x^{1}, & & {\left[X_{0}, X_{1}\right]=\lambda X_{2},} \\
{\left[x^{0}, x^{2}\right]=x^{1}+x^{2},} & & {\left[X_{0}, X_{2}\right]=0,} \\
{\left[x^{1}, x^{2}\right]=0,} & {\left[X_{1}, X_{2}\right]=0 .}
\end{array}
$$

b) Crossed relations:

$$
\begin{array}{llll}
{\left[x^{0}, X_{0}\right]} & =0, & {\left[x^{1}, X_{0}\right]=0,} & {\left[x^{2}, X_{0}\right]=\lambda x^{1},} \\
{\left[x^{0}, X_{1}\right]=-X_{1}-X_{2},} & {\left[x^{1}, X_{1}\right]=\frac{\sinh \left(z X_{0}\right)}{z},} & & {\left[x^{2}, X_{1}\right]=\left(X_{0}-\lambda x^{0}\right) \cosh \left(z X_{0}\right),} \\
{\left[x^{0}, X_{2}\right]=-X_{2},} & {\left[x^{1}, X_{2}\right]=0,} & {\left[x^{2}, X_{2}\right]=\frac{\sinh \left(z X_{0}\right)}{z} .}
\end{array}
$$

\section{Quantum DD algebras: $\mathfrak{g}=\left\{\mathfrak{s}_{3}(\mu), \mathfrak{s}_{3}(0), \mathfrak{n}_{3}\right\}$}

Finally, we shall consider the four remaining cases of DD algebras summarized in Table V. All of them can be fully quantized too.

Table V. DD algebras with $\mathfrak{g}=\left\{\mathfrak{s}_{3}(\mu), \mathfrak{s}_{3}(0), \mathfrak{n}_{3}\right\}$

\begin{tabular}{|c|c|c|c|c|}
\hline$\left(\mathfrak{g}, \mathfrak{g}^{*}\right)$ & $\left(\mathfrak{s}_{3}(\mu), \mathfrak{n}_{3}\right)$ & $\left(\mathfrak{s}_{3}(\mu), \mathfrak{s}_{3}(1 / \mu)\right)$ & $\left(\mathfrak{s}_{3}(0), \mathfrak{n}_{3}\right)$ & $\left(\mathfrak{n}_{3}, \mathfrak{n}_{3}\right)$ \\
\hline $\mathrm{DD}$ & $\mathfrak{s}_{3}(\mu) \odot R^{3}$ & $\mathfrak{s o}(1,3)$ & $\mathfrak{r}_{6}$ & $\mathfrak{n}_{5} \oplus R$ \\
\hline$[\underline{23}$ Nr. & 15 & 16 & $15^{\prime}$ & 17 \\
\hline \hline$\left[x^{0}, x^{1}\right]$ & $\mu x^{1}-x^{2}$ & $\mu x^{1}-x^{2}$ & $-x^{2}$ & 0 \\
\hline$\left[x^{0}, x^{2}\right]$ & $x^{1}+\mu x^{2}$ & $x^{1}+\mu x^{2}$ & $x^{1}$ & 0 \\
\hline$\left[x^{1}, x^{2}\right]$ & 0 & 0 & 0 & $x^{0}$ \\
\hline$\left[X_{0}, X_{1}\right]$ & 0 & $-\lambda X_{1} / \mu+\lambda X_{2}$ & 0 & $\omega X_{2}$ \\
\hline$\left[X_{0}, X_{2}\right]$ & 0 & $-\lambda X_{1}-\lambda X_{2} / \mu$ & 0 & 0 \\
\hline$\left[X_{1}, X_{2}\right]$ & $\mu \omega X_{0}$ & 0 & $\omega X_{0}$ & 0 \\
\hline$\left[x^{0}, X_{0}\right]$ & 0 & 0 & 0 & 0 \\
\hline$\left[x^{0}, X_{1}\right]$ & $\mu \omega x^{2}-\mu X_{1}-X_{2}$ & $-\mu X_{1}-X_{2}$ & $\omega x^{2}-X_{2}$ & 0 \\
\hline$\left[x^{0}, X_{2}\right]$ & $-\mu \omega x^{1}+\mu X_{1}-\mu X_{2}$ & $X_{1}-\mu X_{2}$ & $-\omega x^{1}+X_{1}$ & 0 \\
\hline$\left[x^{1}, X_{0}\right]$ & 0 & $-\lambda x^{1} / \mu-\lambda x^{2}$ & 0 & $-X_{2}$ \\
\hline$\left[x^{1}, X_{1}\right]$ & $\mu X_{0}$ & $\lambda x^{0} / \mu+\mu X_{0}$ & 0 & 0 \\
\hline$\left[x^{1}, X_{2}\right]$ & $-X_{0}$ & $\lambda x^{0}-X_{0}$ & $-X_{0}$ & 0 \\
\hline$\left[x^{2}, X_{0}\right]$ & 0 & $\lambda x^{1}-\lambda x^{2} / \mu$ & 0 & $\omega x^{1}+X_{1}$ \\
\hline$\left[x^{2}, X_{1}\right]$ & $X_{0}$ & $-\lambda x^{0}+X_{0}$ & $X_{0}$ & $-\omega x^{0}$ \\
\hline$\left[x^{2}, X_{2}\right]$ & $\mu X_{0}$ & $\lambda x^{0} / \mu+\mu X_{0}$ & 0 & 0 \\
\hline
\end{tabular}




\subsection{Case $\left(\mathfrak{s}_{3}(\mu), \mathfrak{n}_{3}\right)$}

Coproduct:

$$
\begin{aligned}
\Delta\left(x^{0}\right) & =1 \otimes x^{0}+x^{0} \otimes 1+\mu \omega z\left(x^{1} \otimes x^{2}-x^{2} \otimes x^{1}\right), \\
\Delta\left(x^{1}\right) & =1 \otimes x^{1}+x^{1} \otimes 1, \\
\Delta\left(x^{2}\right) & =1 \otimes x^{2}+x^{2} \otimes 1, \\
\Delta\left(X_{0}\right)= & 1 \otimes X_{0}+X_{0} \otimes 1, \\
\Delta\left(X_{1}\right)= & e^{-\mu z X_{0}} \cos \left(z X_{0}\right) \otimes X_{1}+X_{1} \otimes e^{\mu z X_{0}} \cos \left(z X_{0}\right) \\
& \quad-e^{-\mu z X_{0}} \sin \left(z X_{0}\right) \otimes X_{2}+X_{2} \otimes e^{\mu z X_{0}} \sin \left(z X_{0}\right), \\
\Delta\left(X_{2}\right)= & e^{-\mu z X_{0}} \cos \left(z X_{0}\right) \otimes X_{2}+X_{2} \otimes e^{\mu z X_{0}} \cos \left(z X_{0}\right) \\
& \quad+e^{-\mu z X_{0}} \sin \left(z X_{0}\right) \otimes X_{1}-X_{1} \otimes e^{\mu z X_{0}} \sin \left(z X_{0}\right) .
\end{aligned}
$$

Commutation rules:

a) Subalgebras:

$$
\begin{array}{llrl}
{\left[x^{0}, x^{1}\right]} & =\mu x^{1}-x^{2}, & & {\left[X_{0}, X_{1}\right]=0,} \\
{\left[x^{0}, x^{2}\right]=x^{1}+\mu x^{2},} & {\left[X_{0}, X_{2}\right]=0,} \\
{\left[x^{1}, x^{2}\right]=0,} & {\left[X_{1}, X_{2}\right]=\frac{\omega}{2} \frac{\sinh \left(2 \mu z X_{0}\right)}{z} .}
\end{array}
$$

b) Crossed relations:

$$
\begin{array}{ll}
{\left[x^{0}, X_{0}\right]=0,} & \\
{\left[x^{0}, X_{1}\right]=-\mu X_{1}-X_{2}-\mu \omega x^{1} \sinh \left(\mu z X_{0}\right) \sin \left(z X_{0}\right)+\mu \omega x^{2} \cosh \left(\mu z X_{0}\right) \cos \left(z X_{0}\right),} & {\left[x^{2}, X_{0}\right]=0,} \\
{\left[x^{0}, X_{2}\right]=X_{1}-\mu X_{2}-\mu \omega x^{1} \cosh \left(\mu z X_{0}\right) \cos \left(z X_{0}\right)-\mu \omega x^{2} \sinh \left(\mu z X_{0}\right) \sin \left(z X_{0}\right),} & {\left[x^{2}, X_{1}\right]=\cosh \left(\mu z X_{0}\right) \frac{\sin \left(z X_{0}\right)}{z},} \\
{\left[x^{1}, X_{0}\right]=0,} & {\left[x^{2}, X_{2}\right]=\frac{\sinh \left(\mu z X_{0}\right)}{z} \cos \left(z X_{0}\right) .} \\
{\left[x^{1}, X_{1}\right]=\frac{\sinh \left(\mu z X_{0}\right)}{z} \cos \left(z X_{0}\right),} & \\
{\left[x^{1}, X_{2}\right]=-\cosh \left(\mu z X_{0}\right) \frac{\sin \left(z X_{0}\right)}{z},} &
\end{array}
$$

\subsection{Case $\left(\mathfrak{s}_{3}(\mu), \mathfrak{s}_{3}(1 / \mu)\right)$}

This DD algebra is self-dual for $\mu=1$ and is isomorphic to $\mathfrak{s o}(1,3)$ as an algebra. 


\section{Coproduct:}

$$
\begin{aligned}
& \Delta\left(x^{0}\right)=1 \otimes x^{0}+x^{0} \otimes 1, \\
& \Delta\left(x^{1}\right)=e^{-\frac{\lambda}{\mu} z x^{0}} \cos \left(z \lambda x^{0}\right) \otimes x^{1}+x^{1} \otimes e^{\frac{\lambda}{\mu} z x^{0}} \cos \left(z \lambda x^{0}\right) \\
& -e^{-\frac{\lambda}{\mu} z x^{0}} \sin \left(z \lambda x^{0}\right) \otimes x^{2}+x^{2} \otimes e^{\frac{\lambda}{\mu} z x^{0}} \sin \left(z \lambda x^{0}\right), \\
& \Delta\left(x^{2}\right)=e^{-\frac{\lambda}{\mu} z x^{0}} \cos \left(z \lambda x^{0}\right) \otimes x^{2}+x^{2} \otimes e^{\frac{\lambda}{\mu} z x^{0}} \cos \left(z \lambda x^{0}\right) \\
& +e^{-\frac{\lambda}{\mu} z x^{0}} \sin \left(z \lambda x^{0}\right) \otimes x^{1}-x^{1} \otimes e^{\frac{\lambda}{\mu} z x^{0}} \sin \left(z \lambda x^{0}\right), \\
& \Delta\left(X_{0}\right)=1 \otimes X_{0}+X_{0} \otimes 1, \\
& \Delta\left(X_{1}\right)=e^{-\mu z X_{0}} \cos \left(z X_{0}\right) \otimes X_{1}+X_{1} \otimes e^{\mu z X_{0}} \cos \left(z X_{0}\right) \\
& -e^{-\mu z X_{0}} \sin \left(z X_{0}\right) \otimes X_{2}+X_{2} \otimes e^{\mu z X_{0}} \sin \left(z X_{0}\right), \\
& \Delta\left(X_{2}\right)=e^{-\mu z X_{0}} \cos \left(z X_{0}\right) \otimes X_{2}+X_{2} \otimes e^{\mu z X_{0}} \cos \left(z X_{0}\right) \\
& +e^{-\mu z X_{0}} \sin \left(z X_{0}\right) \otimes X_{1}-X_{1} \otimes e^{\mu z X_{0}} \sin \left(z X_{0}\right) .
\end{aligned}
$$

Commutation rules:

a) Subalgebras:

$$
\begin{array}{llrl}
{\left[x^{0}, x^{1}\right]} & =\mu x^{1}-x^{2}, & {\left[X_{0}, X_{1}\right]=-\frac{\lambda}{\mu} X_{1}+\lambda X_{2},} \\
{\left[x^{0}, x^{2}\right]=x^{1}+\mu x^{2},} & {\left[X_{0}, X_{2}\right]=-\lambda X_{1}-\frac{\lambda}{\mu} X_{2},} \\
{\left[x^{1}, x^{2}\right]=0,} & {\left[X_{1}, X_{2}\right]=0 .}
\end{array}
$$

b) Crossed relations:

$$
\begin{array}{rlrl}
{\left[x^{0}, X_{0}\right]} & =0, & {\left[x^{1}, X_{0}\right]=-\frac{\lambda}{\mu} x^{1}-\lambda x^{2},} \\
{\left[x^{0}, X_{1}\right]=-\mu X_{1}-X_{2},} & {\left[x^{1}, X_{1}\right]=\frac{\sinh \left(z\left(\frac{\lambda}{\mu} x^{0}+\mu X_{0}\right)\right)}{z} \cos \left(z\left(\lambda x^{0}-X_{0}\right)\right),} \\
{\left[x^{0}, X_{2}\right]=X_{1}-\mu X_{2},} & {\left[x^{1}, X_{2}\right]=\frac{\sin \left(z\left(\lambda x^{0}-X_{0}\right)\right)}{z} \cosh \left(z\left(\frac{\lambda}{\mu} x^{0}+\mu X_{0}\right)\right),} \\
{\left[x^{2}, X_{0}\right]=\lambda x^{1}-\frac{\lambda}{\mu} x^{2},} & \\
{\left[x^{2}, X_{1}\right]=-\frac{\sin \left(z\left(\lambda x^{0}-X_{0}\right)\right)}{z} \cosh \left(z\left(\frac{\lambda}{\mu} x^{0}+\mu X_{0}\right)\right),} \\
{\left[x^{2}, X_{2}\right]=\frac{\sinh \left(z\left(\frac{\lambda}{\mu} x^{0}+\mu X_{0}\right)\right)}{z} \cos \left(z\left(\lambda x^{0}-X_{0}\right)\right) .}
\end{array}
$$




\subsection{Case $\left(\mathfrak{s}_{3}(0), \mathfrak{n}_{3}\right)$}

Coproduct:

$$
\begin{aligned}
& \Delta\left(x^{0}\right)=1 \otimes x^{0}+x^{0} \otimes 1+\omega z\left(x^{1} \otimes x^{2}-x^{2} \otimes x^{1}\right), \\
& \Delta\left(x^{1}\right)=1 \otimes x^{1}+x^{1} \otimes 1, \\
& \Delta\left(x^{2}\right)=1 \otimes x^{2}+x^{2} \otimes 1, \\
& \Delta\left(X_{0}\right)=1 \otimes X_{0}+X_{0} \otimes 1, \\
& \Delta\left(X_{1}\right)=\cos \left(z X_{0}\right) \otimes X_{1}+X_{1} \otimes \cos \left(z X_{0}\right)-\sin \left(z X_{0}\right) \otimes X_{2}+X_{2} \otimes \sin \left(z X_{0}\right), \\
& \Delta\left(X_{2}\right)=\cos \left(z X_{0}\right) \otimes X_{2}+X_{2} \otimes \cos \left(z X_{0}\right)+\sin \left(z X_{0}\right) \otimes X_{1}-X_{1} \otimes \sin \left(z X_{0}\right),
\end{aligned}
$$

Commutation rules:

a) Subalgebras:

$$
\begin{array}{ll}
{\left[x^{0}, x^{1}\right]=-x^{2},} & {\left[X_{0}, X_{1}\right]=0,} \\
{\left[x^{0}, x^{2}\right]=x^{1},} & {\left[X_{0}, X_{2}\right]=0,} \\
{\left[x^{1}, x^{2}\right]=0,} & {\left[X_{1}, X_{2}\right]=\omega X_{0} .}
\end{array}
$$

b) Crossed relations:

$$
\begin{aligned}
& {\left[x^{0}, X_{0}\right]=0 \text {, }} \\
& {\left[x^{1}, X_{0}\right]=0,} \\
& {\left[x^{2}, X_{0}\right]=0,} \\
& {\left[x^{0}, X_{1}\right]=-X_{2}+\omega x^{2} \cos \left(z X_{0}\right),} \\
& {\left[x^{1}, X_{1}\right]=0 \text {, }} \\
& {\left[x^{2}, X_{1}\right]=\frac{\sin \left(z X_{0}\right)}{z},} \\
& {\left[x^{0}, X_{2}\right]=X_{1}-\omega x^{1} \cos \left(z X_{0}\right) \text {, }} \\
& {\left[x^{1}, X_{2}\right]=-\frac{\sin \left(z X_{0}\right)}{z} \text {, }} \\
& {\left[x^{2}, X_{2}\right]=0 \text {. }}
\end{aligned}
$$

\subsection{Case $\left(\mathfrak{n}_{3}, \mathfrak{n}_{3}\right)$}

This is an essentially self-dual DD algebra, whose quantization is:

Coproduct:

$$
\begin{aligned}
& \Delta\left(x^{0}\right)=1 \otimes x^{0}+x^{0} \otimes 1 \\
& \Delta\left(x^{1}\right)=1 \otimes x^{1}+x^{1} \otimes 1 \\
& \Delta\left(x^{2}\right)=1 \otimes x^{2}+x^{2} \otimes 1+z \omega\left(x^{0} \otimes x^{1}-x^{1} \otimes x^{0}\right) \\
& \Delta\left(X_{0}\right)=1 \otimes X_{0}+X_{0} \otimes 1-z\left(X_{1} \otimes X_{2}-X_{2} \otimes X_{1}\right) \\
& \Delta\left(X_{1}\right)=1 \otimes X_{1}+X_{1} \otimes 1 \\
& \Delta\left(X_{2}\right)=1 \otimes X_{2}+X_{2} \otimes 1 .
\end{aligned}
$$

a) Subalgebras: 


$$
\begin{array}{lll}
{\left[x^{0}, x^{1}\right]=0,} & {\left[X_{0}, X_{1}\right]=\omega X_{2},} \\
{\left[x^{0}, x^{2}\right]=0,} & {\left[X_{0}, X_{2}\right]=0,} \\
{\left[x^{1}, x^{2}\right]=x^{0},} & {\left[X_{1}, X_{2}\right]=0 .}
\end{array}
$$

b) Crossed relations:

$$
\begin{array}{llll}
{\left[x^{0}, X_{0}\right]=0,} & {\left[x^{1}, X_{0}\right]=-X_{2},} & {\left[x^{2}, X_{0}\right]=X_{1}+\omega x^{1}} \\
{\left[x^{0}, X_{1}\right]=0,} & {\left[x^{1}, X_{1}\right]=0,} & {\left[x^{2}, X_{1}\right]=-\omega x^{0}} \\
{\left[x^{0}, X_{2}\right]=0,} & {\left[x^{1}, X_{2}\right]=0,} & {\left[x^{2}, X_{2}\right]=0 .}
\end{array}
$$

\section{Concluding remarks}

We have presented a comprehensive study of the explicit Hopf algebra quantization of 6D Drinfel'd doubles, thus obtaining a relevant set of new 6D quantum algebras. The only four cases for which we have not succeeded in the quantization belong to the family of classical doubles with $\mathfrak{g}=\mathfrak{r}_{3}(1)$, and could be obtained by applying specific twists on the well-known standard quantization of $\mathfrak{s o}(1,3)$ and $\mathfrak{s l}_{2} \odot R^{3}$ [26]. In general, it becomes apparent that the complexity of the quantum commutation rules that we have obtained is mainly encoded -by construction - in the deformation of the crossed relations $\left[x^{i}, X_{j}\right]$ within the DD algebra.

This study shows that the quantization of the canonical Lie bialgebra structure of a classical double can be directly addressed without making use of the cumbersome construction of the universal $\mathcal{T}$-matrix, which is defined on the canonical dual of the quantum universal enveloping algebra. Obviously, our "analytic" approach does not give any hint concerning the explicit form of the quantum $R$-matrices that should be associated to each of the quantum doubles here constructed. Certainly, this is an open problem.

Finally we stress that, from the point of view of Drinfel'd doubles, non-simple Lie algebras should play a relevant role for the classification of quantum groups.

\section{Acknowledgments}

This work was partially supported by the Ministerio de Educación y Ciencia (Projects BMF2002-02000 and FIS2004-07913), by the Junta de Castilla y León (Projects VA085/02 and BU04/03), and by INFN-CICyT (Italy-Spain).

\section{References}

[1] V.G. Drinfel'd, Sov. Math. Dokl. 27 (1983), 68.

[2] V.G. Drinfel'd, "Quantum Groups" in Proceedings of the International Congress of Mathematicians, Berkeley, 1986, A.M. Gleason (ed.) (AMS, Providence, 1987) 
[3] N. Burroughs, Comm. Math. Phys. 127 (1990) 109

[4] T. Tjin, Int. J. Mod. Phys. A 7 (1992) 6175

[5] M.A. Semenov-Tyan-Shanskii, Theor. Math. Phys. 93 (1992) 1292

[6] S. Majid, J. Geom. Phys. 13 (1994) 169

[7] V. Chari, A. Pressley, A Guide to Quantum Groups, (Cambridge Univ. Press, Cambridge 1994).

[8] S. Majid, Foundations of Quantum Group Theory (Cambridge University Press, Cambridge, 1995)

[9] A. Ballesteros and M.A. del Olmo, "Contractions on the classical double" in Quantum Group Symposium at Group21, H.D. Doebner y V.K. Dobrev (eds.), Heron Press, Sofia, p. 211 (1997)

[10] C. Fronsdal and A. Galindo, Lett. Math. Phys. 27 (1993) 59

[11] M.L. Ge and X.F. Liu, Lett. Math. Phys. 24 (1992) 197

[12] A.A. Vladimirov, Mod. Phys. Lett A. 8 (1993) 2573

[13] C.P. Sun, J. Math. Phys. 34 (1993) 3440

[14] C.P. Sun, W. Li and M.L. Ge, J. Phys. A: Math. Gen. 26 (1993) 5449

[15] C.P. Sun and M.L. Ge, J. Phys. A: Math. Gen. 26 (1993) 7031

[16] D.S. McAnally and I. Tsohantjis, J. Phys. A: Math. Gen. 30 (1997) 651

[17] V.D. Lyakhovsky and V.I. Tkach, J. Phys. A: Math. Gen. 31 (1998) 2869

[18] D. Bernard and A. Leclair, Nucl. Phys. B399 (1993) 709

[19] F.A. Bais and N.M. Muller, Nucl. Phys. B530 (1998) 349

[20] C. Klimcik and P. Severa, Phys. Lett. B351 (1995) 455

[21] M.A. Lledó and V.S. Varadarajan, Lett. Math. Phys. 45 (1998) 247

[22] L. Hlavaty and L. Snobl, Mod. Phys. Lett. A17 (2002) 429

[23] X. Gomez, J. Math. Phys. 41 (2000) 4939

[24] L. Hlavaty and L. Snobl, Int. J. Mod. Phys. A17 (2002) 4043

L. Snobl, J. High Energy Phys. 9 (2002) Art. 018

[25] A. Ballesteros, E. Celeghini and M.A. del Olmo, J. Phys. A: Math. Gen. 37 (2004) 1

[26] A. Ballesteros, F.J. Herranz, M.A. del Olmo and M. Santander J. Phys. A: Math. Gen. 28 (1995) 941

[27] P. Truini and V.S. Varadarajan, Lett. Math. Phys. 24 (1992) 63

P. Truini and V.S. Varadarajan, Lett. Math. Phys. 26 (1992) 53

[28] A. Ballesteros, F.J. Herranz and P. Parashar, J. Phys. A: Math. Gen. 32 (1999) 2369

[29] J. Lukierski, H. Ruegg, V.N. Tolstoy and A. Nowicki, J. Phys. A: Math. Gen. 27 (1994) 2389 